\title{
XMM-Newton study of the spectral variability in NLS1 galaxies
}

\author{
G. Ponti, ${ }^{1,2}$ M. Cappi, ${ }^{2}$ B. Czerny, ${ }^{3}$ R. W. Goosmann ${ }^{4}$ and V. Karas ${ }^{4}$ \\ ${ }^{1}$ Dipartimento di Astronomia, Università degli Studi di Bologna, Via Ranzani 1, \\ I-40127 Bologna, Italy \\ ${ }^{2}$ INAF-IASF Bologna, Via Gobetti 101, I-40129 Bologna, Italy \\ ${ }^{3}$ Copernicus Astronomical Centre, Bartycka 18, P-00 716 Warsaw, Poland \\ ${ }^{4}$ Astronomical Institute, Academy of Sciences, Boční II, CZ-14131 Prague, Czech Republic \\ email: ponti@iasfbo.inaf.it
}

\begin{abstract}
Preliminary results of the study of the X-ray spectral variability of 12 Narrow Line Seyfert 1 (NLS1) galaxies are presented. Rms spectra are calculated and compared for the whole sample to search for possible variations with black hole mass. A larger sample of AGN is under investigation.
\end{abstract}

Keywords. X-rays: galaxies - galaxies: Seyfert - methods: data analysis - black hole physics

We have been studying Root Mean Square (rms) variability of 12 NLS1 galaxies. This sample comprises all NLS1s $\left(\right.$ at $|b| \geqslant 15^{\circ}$ ) detected in the RASS with a PSPC count rate higher than $0.2 \mathrm{c} / \mathrm{s}$ that have been observed with $X M M-N e w t o n$ for more than $30 \mathrm{ks}$ (public data up to July 2006). Figure 1 shows the rms spectra calculated with time bins of a few ks (Ponti et al. 2004). All the sources exhibit significant degree of variability.

Rms spectra have been ordered with increasing black hole mass $M$ from left to right and from top to bottom. All sources with $M<10^{7} M_{\odot}$ show a peak of variability between about 0.5 and $2 \mathrm{keV}$. The decrease of variability at low energy is likely associated to the strong soft excess present in all these sources, the origin of which is highly debated (e.g. Gierliński \& Done 2004). The lower variability at high energy could be due to a pivoting power law (Markowitz \& Edelson 2004; Haardt et al. 1997). Alternatively the whole shape of the rms spectrum could also be due to a variable power law with the presence of either an almost constant ionized disc reflection component (Crummy et al. 2006; Ponti et al. 2006), or a variable absorbing medium (Gierliński \& Done 2006; Chevallier et al. 2006).

For the objects of higher black hole mass the rms spectra become flatter and the variability lower, except for I Zw 1 which shows the maximum of variability at low energy.

\section{References}

Chevallier, L., Collin, S., Dumont, A.-M., Czerny, B., Mouchet, M., Gonçalves, A. C. \& Goosmann, R. 2006, A\&A, 449, 493

Crummy, J., Fabian, A. C., Gallo, L. \& Ross, R. R. 2006, MNRAS, 365, 1067

Gierliński, M. \& Done, C. 2004, MNRAS, 349, L7

Gierliński, M. \& Done, C. 2006, MNRAS, 371, L16

Haardt, F., Maraschi, L. \& Ghisellini, G. 1997, ApJ, 476, 620

Markowitz, A. \& Edelson, R. 2004, ApJ, 617, 939

O'Neill, P. M., Nandra, K., Papadakis, I. E. \& Turner, T. J. 2005, MNRAS, 358, 1405

Ponti, G., Cappi, M., Dadina, M. \& Malaguti, G. 2004, A\&A, 417, 451

Ponti, G., Miniutti, G., Cappi, M., Maraschi, L., Fabian, A. C. \& Iwasawa, K. 2006, MNRAS, 368,903 

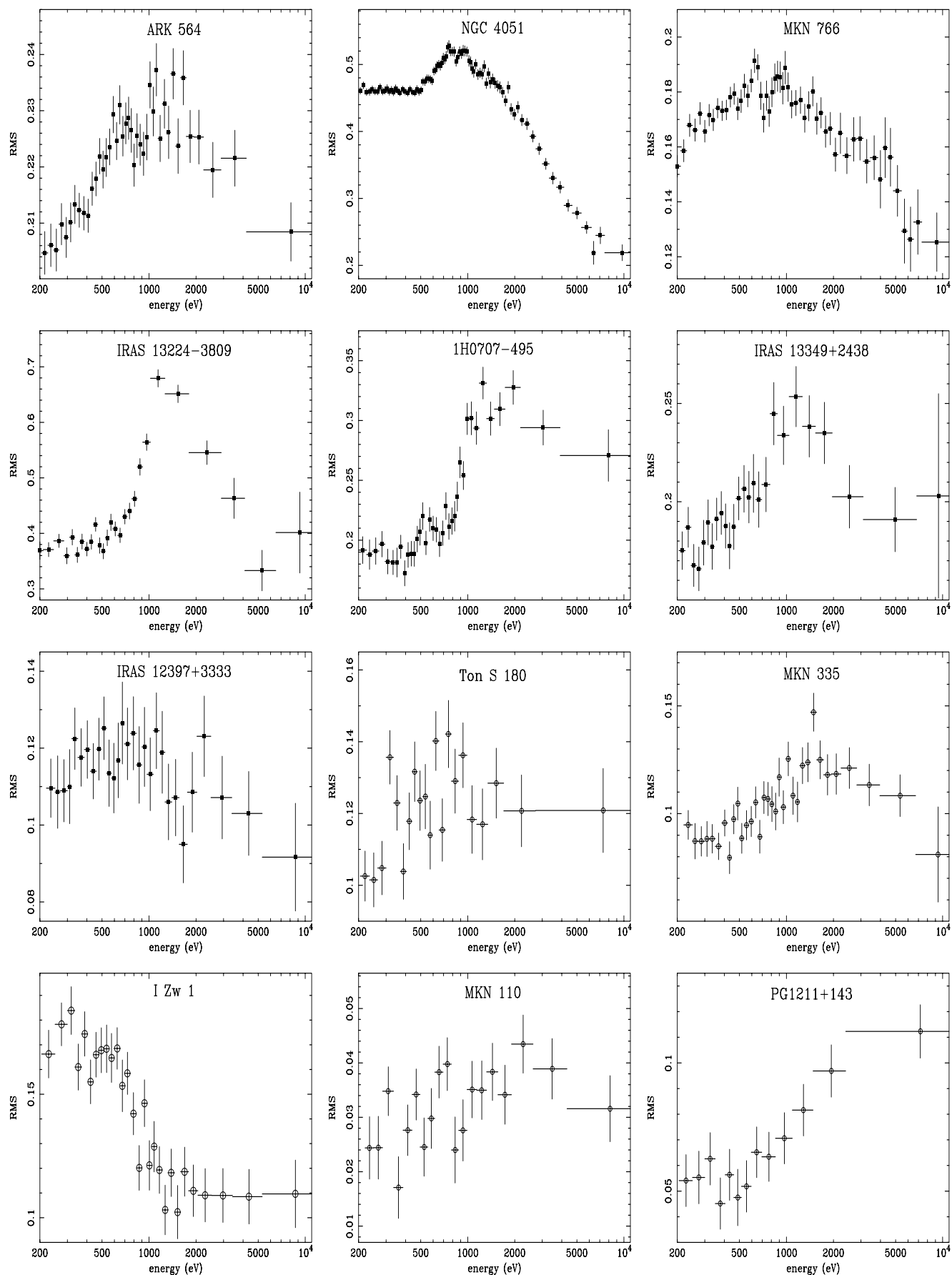

Figure 1. RMS spectra of all the NLS1 of the ROSAT All Sky survey, with a PSPC count rate higher than $0.2 \mathrm{~s}^{-1}$ and a Galactic latitude $|b| \geqslant 15^{\circ}$, observed with XMM-Newton for more than $30 \mathrm{ks}$. The sources are sorted according to the black hole masses: filled squares, open circles, and stars indicate objects with the black hole mass lower than $10^{7} M_{\odot}$, between $10^{7}$ and $10^{8} M_{\odot}$, and higher than $10^{8} M_{\odot}$, respectively. Note: Black hole masses have been all taken from literature (see O'Neill et al. 2005) except for IRAS13224-3809 and 1H0707-495 for which these were assumed based on their strong and fast variability. 